\title{
Basic Study on Surface Insulation Design of Solid/Gas Composite Insulation System with Embedded Electrode
}

\author{
Takahiko Yamashita, Kazuhisa Iwanaga, Tomohiro Furusato \\ Nagasaki University \\ Graduate School of Engineering \\ Nagasaki 852-8521, Japan
}

\author{
Naoki Asari and Junichi Sato \\ Toshiba Corporation Power Systems Company \\ 1, Toshiba-cho, Fuchu-shi \\ Tokyo 183-8511, Japan
}

\begin{abstract}
Solid insulated switchgear (SIS) has been developed as a substitution for medium voltage $\mathrm{SF}_{6}$ gas insulated switchgear. Its main circuits including a vacuum interrupter are coated with epoxy resin and a solid/gas insulation system is formed. For further miniaturization and higher stress design, the authors investigated the surface insulation performance of the solid/gas insulation system containing an embedded electrode. In the previous study, the authors investigated the surface breakdown characteristics. The authors also investigated the surface discharge propagation characteristics and proposed an estimation method of the surface breakdown voltage. In the present study, the authors investigated the surface breakdown characteristics numerically. The calculation was done based on the estimation method proposed previously. The divided voltage ratio, the radius of the embedded electrode, the enhancement factor of the surface insulation strength and the peak value of the surface breakdown voltage are examined. As a result, the characteristics of the parameters and the criteria for the optimum insulation design were clarified.
\end{abstract}

Index Terms - Composite insulation, electric breakdown, simulation, surface discharge, switchgear.

\section{INTRODUCTION}

IN an electric power system, switchgear is used for changing circuit connections. It also takes the role of isolating a fault when a problem occurs in the system. Recently, most of the medium voltage switchgears are cubicle-type low pressure $\mathrm{SF}_{6}$ gas insulated switchgears (C-GIS). However, $\mathrm{SF}_{6}$ gas was designated as one of the targets of emission control in the global warming prevention conference held in 1997 (COP3), then research and development of $\mathrm{SF}_{6}$ gas free systems were actively advanced [1-3].

Regarding the situation mentioned above, a solid insulated switchgear (SIS), in which main circuits including a vacuum interrupter are coated with epoxy resin, has been developed [2]. However, improvement of the surface insulation performance of the solid/gas composite insulation system is required for the development of higher stress and compact design. Therefore, the authors investigated on the insulation performance using test samples which are the model of the moving part of SIS. The test sample was a disc-shaped insulation plate of epoxy resin in which a metal electrode was embedded.

Manuscript received 21 March 2017, in final form 11 May 2017, accepted 12 May 2017. Corresponding author: T. Yamashita.
In the previous study [4], the authors measured the surface breakdown voltage (BDV) and the partial discharge inception voltage (PDIV) under ac or impulse voltage application. The measurement was done by changing the radius of the embedded electrode $\left(r_{\mathrm{e}}\right)$, thickness of the disc-shaped epoxy resin plate $(t)$ or the radius of the epoxy resin plate $\left(r_{\mathrm{p}}\right)$. The results indicated that it was possible to improve the surface insulation characteristics by choosing the radius of the embedded electrode appropriately.

Furthermore, the authors investigated the estimation of the surface breakdown voltage. In the previous paper [5], the authors measured the surface discharge propagation characteristics experimentally and proposed an estimation method of BDV based on the surface discharge propagation characteristics. From the comparison between the estimated value and the measured value in [4], a good correlation was obtained.

In the present study, the surface breakdown characteristics were calculated using the estimation method proposed in the previous paper [5]. The divided voltage ratio, the radius of the embedded electrode, the enhancement factor of the surface insulation strength and the peak value of the surface breakdown 
voltage are examined. As a result, the characteristics of the parameters and the criteria for the optimum insulation design were clarified. The authors believe that the results obtained in the present study are helpful for the design of the solid/gas composite insulation system.

\section{SIMULATION MODEL}

Figure 1 shows the cross section of a sample used for the simulation of the breakdown characteristics of the solid/gas composite insulation system. This sample is the model of insulation system of SIS and is composed of a disc-shaped epoxy resin plate with back-side electrode and a disc-shaped embedded electrode. The thickness of the embedded electrode is $2 \mathrm{~mm}$ and its radius of curvature at the edge is $1 \mathrm{~mm}$. The distance between the bottom of the embedded electrode and the back-side electrode is $4 \mathrm{~mm}$. The embedded electrode is connected to the center rod electrode with a radius of $5 \mathrm{~mm}$.

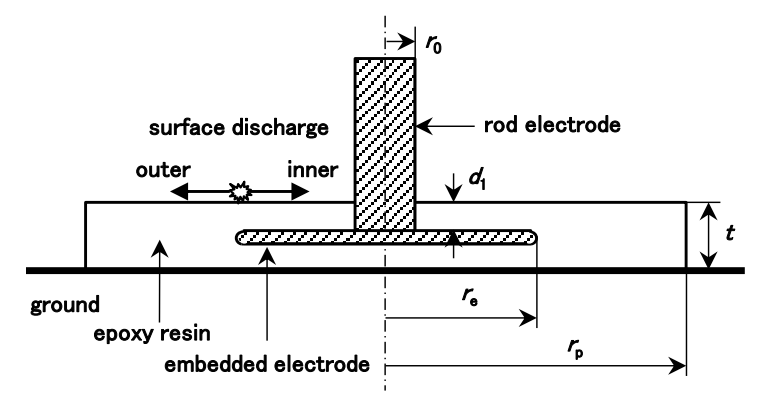

Figure 1. A model of composite insulation system with embedded electrode used for the simulation. $r_{\mathrm{p}}$ is the radius of the disc-type epoxy resin plate, $r_{\mathrm{e}}$ is the radius of the embedded electrode, $t$ is the thickness of the epoxy resin plate, $r_{0}$ is the radius of the center rod electrode and $d_{1}$ is the thickness of the epoxy resin between the surface of the plate and the embedded electrode.

In the previous study [4], the surface discharge was revealed to initiate at the position on the surface just above the edge of the embedded electrode. The surface discharge propagates in both direction towards the center rod electrode and towards the ground electrode. The surface breakdown takes place when both of the surface discharges reach the opposite electrodes. In the previous study [5], the surface breakdown voltage was estimated based on the propagation characteristics of the surface discharge. In the present study, the surface breakdown voltage was also calculated based on the estimation method proposed in the previous study [5]. In addition, potential distribution along the surface was calculated using a software, Ansoft Maxwell (Ansoft Japan Co., Ltd.).

\section{SURFACE BREAKDOWN VOLTAGE}

In the previous paper [5], the surface breakdown voltage (BDV) under impulse voltage application was found to depend on the polarity. However, lower value should be taken as the impulse BDV. In the estimation, the surface breakdown voltage is determined by comparing the flashover characteristics between the surface discharges propagate inner and outer surface from the point of the maximum field strength along the surface. The surface flashover voltage of positive polarity was found lower than that of negative. Therefore, impulse BDV is determined by the positive surface flashover characteristics.

The surface flashover voltage under positive impulse application $\left(V_{\text {pos. }}\right)$ was found as follows [5]

$$
V_{\text {pos. }}=K_{\text {pos. }} l^{\frac{1}{4}} C_{\mathrm{b}}^{-\frac{3}{8}}
$$

where $l(\mathrm{~m})$ was the surface length, $C_{\mathrm{b}}\left(\mathrm{F} / \mathrm{m}^{2}\right)$ was the distributed electrostatic capacity per unit area and $\mathrm{K}_{\text {pos. }}$ was a constant which was 57.56. From the comparison between the values obtained from the following equations [5], the surface breakdown voltage is determined as the lower value.

$$
\begin{aligned}
& V_{\mathrm{F}_{\text {in. }}}=\left(\frac{1}{1-D}\right) V_{\text {pos. }} \quad\left(C_{\mathrm{b}}=C_{\mathrm{b}}^{\prime}, l=l_{\text {in. }}\right) \\
& V_{\mathrm{F}_{\text {out. }}}=\frac{1}{D} V_{\text {pos. }} \quad\left(l=l_{\text {out. }}\right)
\end{aligned}
$$

where $V_{\mathrm{F}_{\text {in }} \text {. }}$ and $V_{\mathrm{F}_{\text {out. }}}$ are the flashover voltages of the inner and outer surfaces, respectively. $D$ is the divided voltage ratio which is obtained from the voltage across the outer surface divided by the full voltage between the center rod electrode and the ground. $C_{\mathrm{b}}^{\prime}$ is the distributed electrostatic capacity per unit area for the inner part. Then, $C_{\mathrm{b}}=C_{\mathrm{b}}^{\prime}$ means that the thickness of the dielectric of the inner part is different from the thickness of the

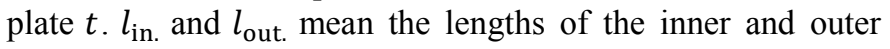
surfaces, respectively.

Figure 2 shows the impulse breakdown voltage (BDV) under the conditions where the thickness of the epoxy resin plate $t$ is $11 \mathrm{~mm}$ and the radius of the plate $r_{\mathrm{p}}$ is $100 \mathrm{~mm}$. In Figure 2, measured values of impulse BDV are also shown. Owing to the

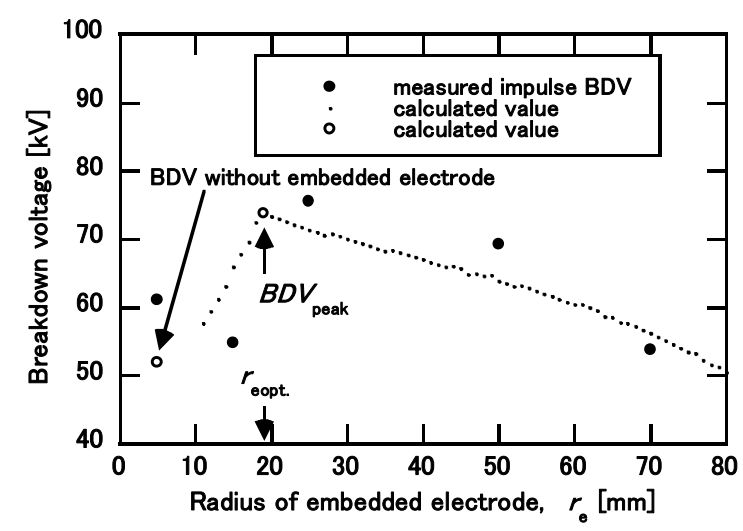

Figure 2. Relation between estimated impulse breakdown voltage and the radius of the embedded electrode. $t=11(\mathrm{~mm})$ and $r_{\mathrm{p}}=100(\mathrm{~mm})$.

polarity effect, lower value was selected for each experimental condition.

Equations (2) and (3) are also applied to ac breakdown voltage. Therefore, the calculated characteristic of the impulse BDV shown in Figure 2 is found to be the same characteristic of ac BDV. The calculated characteristic is found to agree well with the measured impulse BDV [4].

In Figure 2, it is found that BDV has a peak value ( $\left.B D V_{\text {peak }}\right)$. The radius of the embedded electrode corresponding to $B D V_{\text {peak }}$ is expressed as $r_{\mathrm{e}_{\text {opt. }}}$. They will be discussed in the following section. 


\subsection{OPTIMUM RADIUS OF EMBEDDED ELECTRODE}

Figure 3 shows the relation between the position of the maximum field strength and the radius of the embedded electrode when the thickness of the epoxy resin plate $t$ was 11 $\mathrm{mm}$ and the radius of the plate was $100 \mathrm{~mm}$ as an example. As

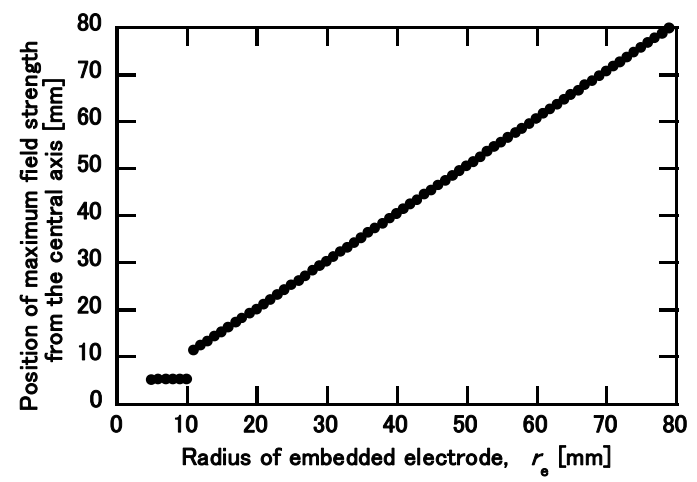

Figure 3. An example of the relation between the position of the maximum field strength and the radius of the embedded electrode. $t=11(\mathrm{~mm})$ and $r_{\mathrm{p}}=100(\mathrm{~mm})$.

shown in Figure 3, although the position of the maximum field strength exists on the surface of the center rod electrode in the small $r_{\mathrm{e}}$ region, the position of the maximum field strength is almost the same as $r_{\mathrm{e}}$ except for the small $r_{\mathrm{e}}$ region. In other cases, similar results were obtained. BDV will be discussed except for the small $r_{\mathrm{e}}$ region. Then, the authors assume that the position of maximum field strength is the same as $r_{\mathrm{e}}$.

From the assumption, equations (2) and (3) are re-written using equation (1) and the radius of the center rod electrode $r_{0}$. In the following equations, $r_{0}, r_{\mathrm{e}}$ and $r_{\mathrm{p}}$ are the values in meters.

$$
\begin{aligned}
& V_{\mathrm{F}_{\text {in. }}}=\frac{1}{1-D}\left\{\mathrm{~K}_{\text {pos. }} l^{\frac{1}{4}} C^{\prime}{ }_{\mathrm{b}}^{-\frac{3}{8}}\right\} \\
& =\frac{1}{1-D}\left\{\mathrm{~K}_{\text {pos. }}\left(r_{\mathrm{e}}-r_{0}\right)^{\frac{1}{4}} C_{\mathrm{b}}^{\prime}-\frac{3}{8}\right\} \\
& V_{\mathrm{F}_{\text {out. }}}=\frac{1}{D}\left\{\mathrm{~K}_{\text {pos. }} l \frac{1}{4} C_{\mathrm{b}}^{-\frac{3}{8}}\right\} \\
& =\frac{1}{D}\left\{\mathrm{~K}_{\text {pos. }}\left(r_{\mathrm{p}}-r_{\mathrm{e}}\right)^{\frac{1}{4}} C_{\mathrm{b}}^{-\frac{3}{8}}\right\}
\end{aligned}
$$

When the thickness of the epoxy resin plate and the radius of the plate are given, the radius of the embedded electrode when BDV has a peak is obtained from the equations (2) and (3). $\mathrm{BDV}$ takes a peak under the condition where $V_{\mathrm{F}_{\text {in. }}}=V_{\mathrm{F}_{\text {out }}}$.

$$
r_{\mathrm{e}_{\text {opt. }}}=\frac{\left(\frac{1-D}{D}\right)^{4}\left(\frac{C^{\prime} \mathrm{b}}{C_{\mathrm{b}}}\right)^{1.5} r_{\mathrm{p}}+r_{0}}{1+\left(\frac{1-D}{D}\right)^{4}\left(\frac{C^{\prime} \mathrm{b}}{C_{\mathrm{b}}}\right)^{1.5}}
$$

Here, the authors define $r_{\mathrm{e}_{\text {opt. }}}$ as the optimum $r_{\mathrm{e}}$. Using the thickness of the dielectric, $r_{\mathrm{e}}$ opt. following equation (8). In general, the distributed electrostatic capacity per unit area $C\left(\mathrm{~F} / \mathrm{m}^{2}\right)$ is expressed with the following equation (7).

$$
C=\frac{\varepsilon_{\mathrm{r}} \times 8.854 \times 10^{-12}}{d}
$$

where $\varepsilon_{\mathrm{r}}$ is the relative permittivity of the dielectric and $d(\mathrm{~m})$ is the thickness of the dielectric.

$$
r_{\mathrm{e}_{\mathrm{opt}}}=\frac{\left(\frac{1-D}{D}\right)^{4}\left(\frac{d_{2}}{d_{1}}\right)^{1.5} r_{\mathrm{p}}+r_{0}}{1+\left(\frac{1-D}{D}\right)^{4}\left(\frac{d_{2}}{d_{1}}\right)^{1.5}}
$$

where $d_{1}(\mathrm{~m})$ is the thickness of the dielectric of the inner part and $d_{2}(\mathrm{~m})$ is the thickness of the dielectric of the outer part.

These equations suggest that the optimum radius of the embedded electrode $r_{\mathrm{e}_{\text {opt. }}}$ is determined uniquely by the radius of the epoxy resin plate $r_{\mathrm{p}}$ and the thickness of the plate $t$ under the present conditions where the radius of the center rod electrode $r_{0}$ and the distance between the bottom of the embedded electrode and the back-side electrode are given.

\subsection{PEAK VALUE OF BDV}

From the above equations, the peak value of the surface breakdown voltage ( $B D V_{\text {peak }}$ ) for given thickness of the epoxy resin plate $t$ and the radius of the plate $r_{\mathrm{p}}$ is obtained as follows. In the following equation, $r_{0}$ and $r_{\mathrm{p}}$ are the values in meters.

$$
\begin{aligned}
& B D V_{\text {peak }}= \\
& \frac{1}{1-D} \mathrm{~K}_{\text {pos. }}\left\{\frac{\left(\frac{1-D}{D}\right)^{4}\left(\frac{C_{\mathrm{b}}^{\prime}}{C_{\mathrm{b}}}\right)^{1.5} r_{\mathrm{p}}+r_{0}}{1+\left(\frac{1-D}{D}\right)^{4}\left(\frac{C^{\prime} \mathrm{b}}{C_{\mathrm{b}}}\right)^{1.5}}-r_{0}\right\}^{\frac{1}{4}} C_{\mathrm{b}}^{\prime}{ }^{-\frac{3}{8}} \\
& =\mathrm{K}_{\text {pos. }}\left(r_{\mathrm{p}}-r_{0}\right)^{\frac{1}{4}} C_{\mathrm{b}}{ }^{-\frac{3}{8}} \times \\
& \left.=\mathrm{K}_{\text {pos. }}\left(r_{\mathrm{p}}-r_{0}\right)^{\frac{1}{4}} C_{\mathrm{b}}{ }^{-\frac{3}{8}} \times(1-D)^{4}\left(\frac{C_{\mathrm{b}}^{\prime}}{C_{\mathrm{b}}}\right)^{1.5}\right\}^{-\frac{1}{4}} \\
& \quad\left\{D^{4}+(1-D)^{4}\left(\frac{d_{2}}{d_{1}}\right)^{1.5}\right\}^{-\frac{1}{4}} \\
& =F_{\mathrm{e}} \times \mathrm{K}_{\text {pos. }}\left(r_{\mathrm{p}}-r_{0}\right)^{\frac{1}{4}} C_{\mathrm{b}}{ }^{-\frac{3}{8}} \\
& F_{\mathrm{e}}=\left\{D^{4}+(1-D)^{4}\left(\frac{d_{2}}{d_{1}}\right)^{1.5}\right\}^{-\frac{1}{4}}
\end{aligned}
$$

The part $\mathrm{K}_{\text {pos. }}\left(r_{\mathrm{p}}-r_{0}\right)^{\frac{1}{4}} C_{\mathrm{b}}{ }^{-\frac{3}{8}}$ corresponds to the positive surface flashover voltage under the conditions where the surface distance is $r_{\mathrm{p}}-r_{0}$ and the embedded electrode has nooverhang. Therefore, $F_{\mathrm{e}}$ is defined as a enhancement factor of the surface insulation strength. $F_{\mathrm{e}}$ means the effect of embedding electrode on the characteristics of the surface insulation performance.

\section{CHARACTERISTICS OF PARAMETERS 4.1 DIVIDED VOLTAGE RATIO}

Figure 4 shows an example of the relation between the divided voltage ratio $D$ and the radius of the embedded electrode $r_{\mathrm{e}}$ when the thickness of the epoxy resin plate $t$ is 11 $\mathrm{mm}$ and the radius of the plate $r_{\mathrm{p}}$ is $100 \mathrm{~mm}$. As shown in Figure $4, D$ is around 0.7 except for the small $r_{\mathrm{e}}$ region. In that region, $D$ is 1 and the position of the maximum field strength exists on the center rod electrode. The region is not suitable for 


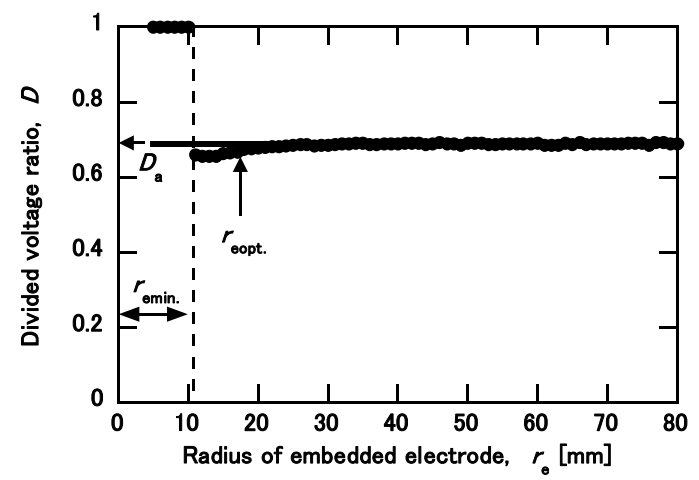

Figure 4. An example of the variation of the voltage divided ratio with the radius of the embedded electrode. $t=11(\mathrm{~mm})$ and $r_{\mathrm{p}}=100(\mathrm{~mm})$.

the optimum surface insulation design. Therefore, it is deduced that the radius of the embedded electrode $r_{\mathrm{e}}$ has the minimum value. The characteristics of the minimum radius of the embedded electrode $r_{\mathrm{e}}$ min. . will be discussed in the following section.

In order to discuss the relation between the divided voltage ratio $D$ and the radius of the epoxy resin plate $r$ or the relation between the divided voltage ratio $D$ and the thickness of the plate $t$, the authors made an average of $D$ according to the procedure which is explained below. In that region, there was a little dispersion in $D$. It was supposed to be caused by the accuracy of the numerical calculation and was found less than $4 \%$. In other cases, similar results were obtained. Then, the authors made an average of $D$ in the region in which the deviation becomes less than $\pm 3 \%$. The average is used as a representative value of $D$ independent of $r_{\mathrm{e}}$ and is expressed with $D_{\mathrm{a}}$. Then, $D_{\mathrm{a}}$ was calculated under the conditions where the thickness of the epoxy resin between the embedded electrode and the ground was $4 \mathrm{~mm}$, the radius of the embedded electrode $r_{\mathrm{p}}$ was varied in the range from 100 to $300 \mathrm{~mm}$ and the thickness of the epoxy resin plate $t$ was varied in the range from 10 to $40 \mathrm{~mm}$.

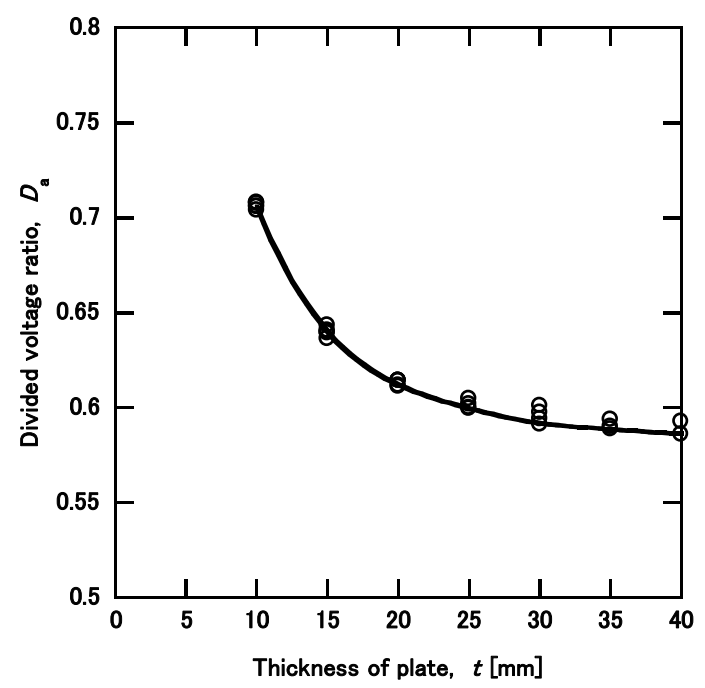

Figure 5. Relation between $D_{\mathrm{a}}$ and the thickness of the epoxy resin plate $t$.
The calculation results are shown in Figure 5. The figure shows the relation between $D_{\mathrm{a}}$ and the thickness of the epoxy resin plate $t$. In Figure 5, $D_{\mathrm{a}}$ for given $t$ is not classified by $r_{\mathrm{p}}$. Although there are quite small differences in $D_{\mathrm{a}}$ for given $t, D_{\mathrm{a}}$ is mostly constant for given $t$. Therefore, it is deduced that $D_{\mathrm{a}}$ is a function of the thickness of the plate $t$ regardless of the radius of the plate $r_{\mathrm{p}} . D_{\mathrm{a}}$ decreases with $t$ and seemed to be saturated.

From the above, the divided voltage ratio was found to vary with the thickness of the epoxy resin plate and to be almost independent of the radius of the embedded electrode or the radius of the epoxy resin plate. In general, the divided voltage ratio is decided by the ratio between the voltages across the solid dielectric material and the air gap. The relative permittivity of the solid insulation material is usually larger than that of air and the air gap length in the present configurations is usually larger than the thickness of the solid insulation material. Therefore, the divided voltage ratio is supposed to be strongly affected by the thickness of the epoxy resin between the embedded electrode and the surface.

In the present study, the thickness of the epoxy resin between the embedded electrode and the ground is $4 \mathrm{~mm}$ and the thickness of the embedded electrode is $2 \mathrm{~mm}$. Then, the thickness of the epoxy resin between the embedded electrode and the surface becomes $(t-6) \mathrm{mm}$. When the thickness of the epoxy resin between the embedded electrode and the surface is given, the divided voltage ratio can be decided approximately from Figure 5.

\subsection{ENHANCEMENT FACTOR OF SURFACE INSULATION STRENGTH}

From equation (10), the enhancement factor of the surface insulation strength $F_{\mathrm{r}}$ is re-written using $D_{\mathrm{a}}$.

$$
F_{\mathrm{r}} \approx\left\{D_{\mathrm{a}}{ }^{4}+\left(1-D_{\mathrm{a}}\right)^{4}\left(\frac{d_{2}}{d_{1}}\right)^{1.5}\right\}^{-\frac{1}{4}}
$$

$D_{\mathrm{a}}$ depends on the thickness of the plate $t$, then $F_{\mathrm{r}}$ also depends on $t$.

The enhancement factor of the surface insulation strength $F_{\mathrm{r}}$ was calculated using $D_{\mathrm{a}}$ under the conditions where the thickness of the epoxy resin between the embedded electrode and the ground was $4 \mathrm{~mm}$, the radius of the embedded electrode $r_{\mathrm{p}}$ was varied in the range from 100 to $300 \mathrm{~mm}$ and the thickness of the epoxy resin plate $t$ was varied in the range from 10 to $40 \mathrm{~mm}$.

The calculation results are shown in Figure 6. The figure shows the relation between $F_{\mathrm{e}}$ and the thickness of the epoxy resin plate $t$. Similar to the case of $D_{\mathrm{a}}, F_{\mathrm{e}}$ for given $t$ is not classified by $r_{\mathrm{p}}$. Although there are quite small differences in $F_{\mathrm{e}}$ for given $t, F_{\mathrm{e}}$ is mostly constant for given $t$. Therefore, it is deduced that $F_{\mathrm{e}}$ is a function of the thickness of the plate $t$ regardless of the radius of the plate $r_{\mathrm{p}} . F_{\mathrm{e}}$ decreases with the thickness of the plate $t$ and seemed to be saturated.

From equation (11), when the thickness of the plate $t$ is given, $\frac{d_{2}}{d_{1}}$ is constant under the present condition and $D_{\mathrm{a}}$ becomes almost constant regardless of the radius of the plate $r_{\mathrm{p}}$, as shown in Figure 5. Then, $F_{\mathrm{e}}$ becomes almost constant regardless of the 


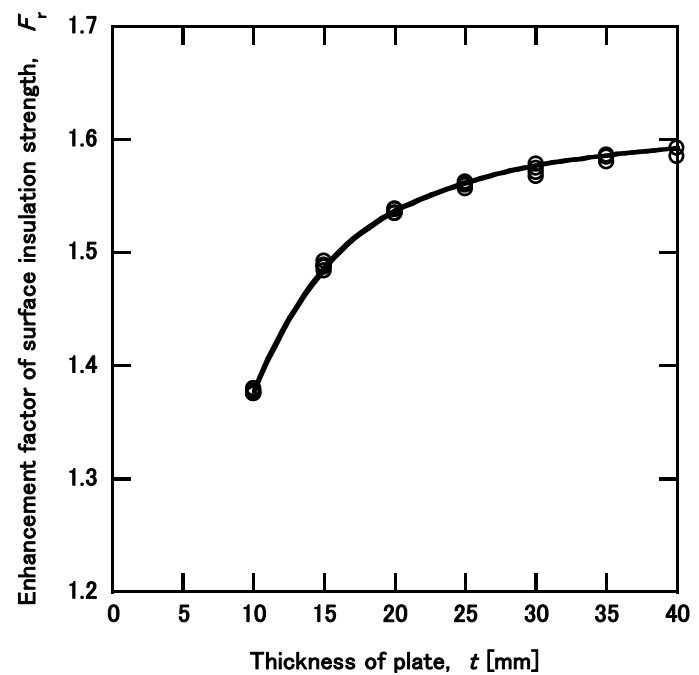

Figure 6. Relation between the enhancement factor of surface insulation strength and the thickness of the plate.

radius of the plate $r_{\mathrm{p}}$ and varies with the thickness of the plate $t$. Therefore, when the thickness of the epoxy resin between the embedded electrode and the surface is given, $F_{\mathrm{e}}$ can be decided from Figure 6, approximately.

\subsection{RADIUS OF EMBEDDED ELECTRODE}

At first, the minimum radius of the embedded electrode mentioned above was examined. The minimum radius of the embedded electrode $r_{\mathrm{e}_{\min } \text {. }}$ was obtained from the calculation of the potential distribution along the surface under the conditions where the thickness of the epoxy resin between the embedded electrode and the ground was $4 \mathrm{~mm}$, the radius of the embedded electrode $r_{\mathrm{p}}$ was varied in the range from 100 to $300 \mathrm{~mm}$ and the thickness of the epoxy resin plate $t$ was varied in the range from 10 to $40 \mathrm{~mm}$.

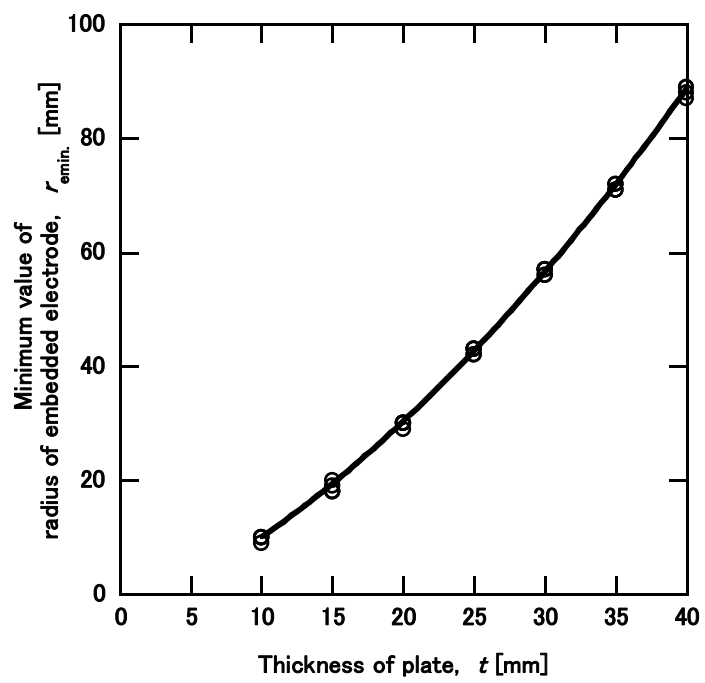

Figure 7. The minimum value of the radius of the embedded electrode $r_{\mathrm{e}_{\text {min. }}}$ for given thickness of the plate $t$.
The calculation results are shown in Figure 7. The figure shows the relation between the minimum radius of the

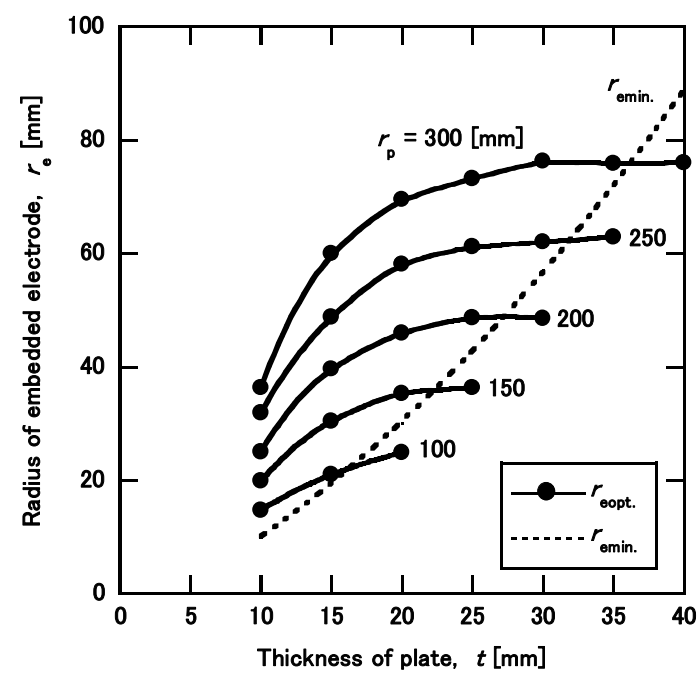

Figure 8. Relation between the optimum radius of the embedded electrode $r_{\mathrm{e}_{\text {opt. }}}$ and the thickness of the plate $t$ compared with the minimum value of the radius of the embedded electrode $r_{\mathrm{e}_{\min } \text {. }}$.

embedded electrode $r_{\mathrm{e}}{ }_{\min }$ and the thickness of the epoxy resin plate $t$. As a result, it is found that $r_{\mathrm{e}_{\text {min. }}}$ for given thickness of the epoxy resin plate $t$ is almost constant regardless of the radius of the plate $r_{\mathrm{p}}$. Therefore, it is deduced that $r_{\mathrm{e}_{\min } \text {. is a }}$ function of the thickness of the plate $t$ regardless of the radius of the plate $r_{\mathrm{p}}$. The minimum value of the radius of the embedded electrode $r_{\mathrm{e}}$ min. increases with the thickness of the plate $t$.

The value of BDV in the case where the position of the maximum field strength exists on the surface of the center rod electrode is supposed to be similar to that in the case where the embedded electrode has no-overhang. That is, $F_{\mathrm{e}}=1$ in equation (9) and the effect of embedding an electrode on the improvement of the insulation performance is not expected. Therefore, the region is not suitable for the insulation design. From this, the radius of the embedded electrode $r_{\mathrm{e}}$ should be larger than the minimum radius of the embedded electrode $r_{\mathrm{e}_{\text {min }} \text {. }}$ for the optimum insulation design.

The optimum radius of the embedded electrode $r_{\mathrm{e}_{\mathrm{opt}}}$. expressed by equation (8) is re-written using $D_{\mathrm{a}}$.

$$
r_{\mathrm{e}_{\mathrm{opt}}} \approx \frac{\left(\frac{1-D_{a}}{D_{a}}\right)^{4}\left(\frac{d_{2}}{d_{1}}\right)^{1.5} r_{\mathrm{p}}+r_{0}}{1+\left(\frac{1-D_{a}}{D_{a}}\right)^{4}\left(\frac{d_{2}}{d_{1}}\right)^{1.5}}
$$

In the present condition, the thickness of the embedded electrode and the distance between the bottom of the embedded electrode and the ground $\left(d_{2}-d_{1}=t-d_{1}\right)$ are constants. When the thickness of the epoxy resin plate $t=d_{2}$ is given, $D_{\mathrm{a}}$ becomes a function of $t$ as mentioned in Section 4.1. $r_{\mathrm{e}_{\text {opt. }}}$ was calculated using equation (12).

The calculation results are shown in Figure 8. The figure shows the relation between the optimum radius of the 
embedded electrode $r_{\mathrm{e}_{\text {opt. }}}$ and the thickness of the plate $t$ as a parameter of the radius of the plate $r_{\mathrm{p}}$. In Figure 8, the minimum radius of the embedded electrode $r_{\mathrm{e}_{\text {min. }}}$ in Figure 7 is also shown.

As mentioned above, the radius of the embedded electrode should be larger than the minimum radius of the embedded electrode for the optimum insulation design. Therefore, the optimum radius of the embedded electrode $r_{\mathrm{e}_{\text {opt }}}$ should be larger than the minimum radius of the embedded electrode $r_{\mathrm{e}_{\min }}$.

$$
r_{\mathrm{e}_{\mathrm{opt}}} \geq r_{\mathrm{e}_{\min }}
$$

Equation (13) shows one of the design criteria for the optimum insulation design.

From the comparison between $r_{\mathrm{e}_{\mathrm{opt}}}$ and $r_{\mathrm{e}_{\min } \text {. }}$ in Figure 8, the limits for the optimum insulation design can be found. The intersection points indicate the minimum values of $r_{\mathrm{e}_{\mathrm{opt}}}$ for given thickness of the plate $t$. That is, they indicate the existence of the minimum values of $r_{\mathrm{p}}$ for given $t$. In other words, the maximum value of the thickness of the plate exists for given radius of the plate.

\subsection{PEAK VALUE OF SURFACE BREAKDOWN VOLTAGE}

Peak value of the surface breakdown voltage $B D V_{\text {peak }}$ was calculated by the following equation.

$$
\begin{aligned}
& B D V_{\text {peak }} \approx\left\{D_{\mathrm{a}}{ }^{4}+\left(1-D_{\mathrm{a}}\right)^{4}\left(\frac{d_{2}}{d_{1}}\right)^{1.5}\right\}^{-\frac{1}{4}} \\
& \times \mathrm{K}_{\text {pos. }}\left(r_{\mathrm{p}}-r_{0}\right)^{\frac{1}{4}} C_{\mathrm{b}}{ }^{-\frac{3}{8}}
\end{aligned}
$$

The calculation was conducted for given radius of the epoxy resin plate $r_{\mathrm{p}}$ (in equation (14), $r_{\mathrm{p}}$ is expressed in meters). The calculation results are shown in Figure 9. In the figure, the relation between the calculated $B D V_{\text {peak }}$ and the thickness of the plate $t$ as a parameter of the radius of the plate $r_{\mathrm{p}}$ are shown.

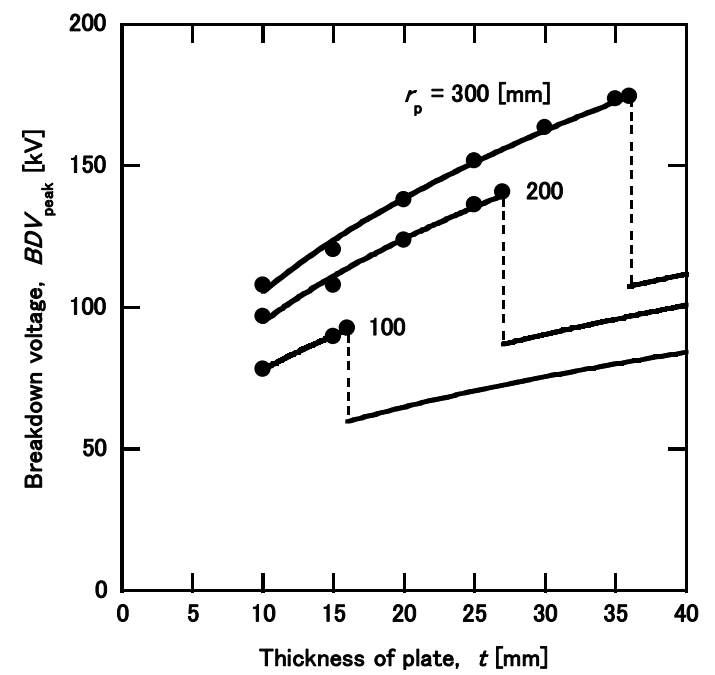

Figure 9. Relation between the peak value of the impulse breakdown voltage $B D V_{\text {peak }}$ and the thickness of the plate $t . r_{\mathrm{p}}=100,200$ and $300(\mathrm{~mm})$.
For given $r_{\mathrm{p}}, B D V_{\text {peak }}$ increases with $t$. However, $B D V_{\text {peak }}$ becomes quite low when the thickness of the plate $t$ became larger than the value obtained from the intersection point in Figure 8. In the region where $B D V_{\text {peak }}$ becomes low, the surface discharge will start from the center rod electrode. That means that the enhancement factor of the surface insulation strength $F_{\mathrm{e}}$ (equation (10)) becomes 1 . Therefore, $B D V_{\text {peak }}$ becomes quite low and gradually increases with $t$.

The breakdown voltage in the small $t$ region is suitable for the insulation design and the maximum value of the surface breakdown voltage in this region becomes critical. Therefore, the value of the thickness of the plate at the intersection point gives the maximum thickness of the plate.

\section{CRITERIA FOR INSULATION DESIGN}

From the above discussion, the combination of the minimum radius and the maximum thickness of the epoxy resin plate gives the critical condition. Under the critical condition, the peak value of the surface breakdown voltage has the critical value. In this section, the relations among the critical breakdown voltage, the minimum radius of the epoxy resin plate and the maximum thickness of the plate are summarized and the criteria for the optimum insulation design are discussed.

\subsection{MINIMUM RADIUS AND MAXIMUM THICKNESS OF PLATE}

As pointed out in the former section, the minimum radius $r_{\mathrm{p}_{\text {min. }}}$ and the maximum thickness $t_{\max }$ of the epoxy resin plate exists for the optimum insulation design. The combination of the minimum radius $r_{\mathrm{p}_{\text {min. }}}$ and the maximum thickness $t_{\max }$ (critical condition) can be derived from Figure 9.

The critical condition is shown in Figure 10, as the relation between the minimum radius $r_{\mathrm{p}_{\min }}$ and the maximum thickness $t_{\text {max. }}$ of the plate. The curve in Figure 10 shows the critical condition.

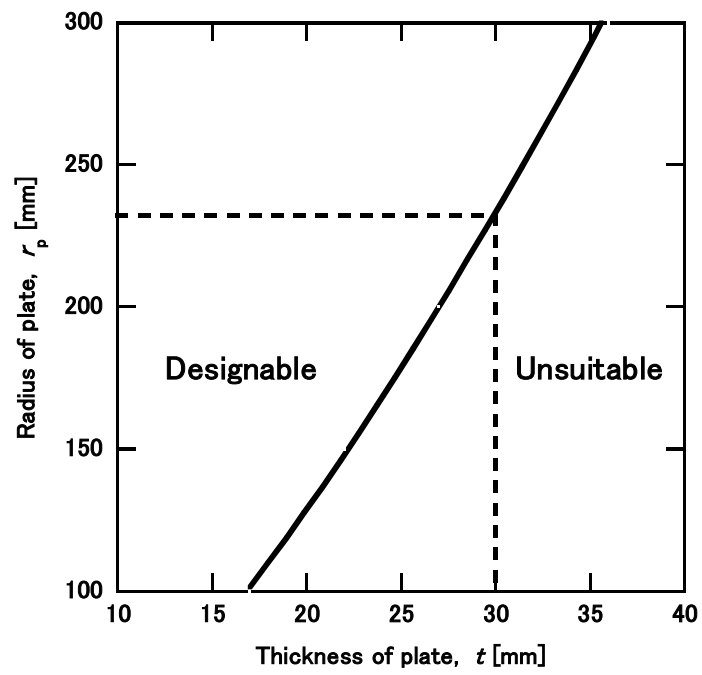

Figure 10. Critical conditions of the radius and the thickness of the plate: the relation between the minimum radius and the maximum thickness of the plate. Broken lines indicate an example when lightning impulse withstand voltage (LIWV) of $150 \mathrm{kV}$ is given. Radius of embedded electrode is determined from Figure 11. The details are mentioned in Section 5.2. 


\subsection{SURFACE BREAKDOWN VOLTAGE}

From the critical condition in Figure 10, the critical value of the surface breakdown voltage $B D V_{\text {peak }}$ (crit.) is calculated using the combination of the minimum radius $r_{\mathrm{p}_{\text {min }} \text {. }}$ and the maximum thickness $t_{\max }$ and the following equations.

$$
\begin{aligned}
& B D V_{\text {peak }}(\text { crit. })= \\
& F_{\mathrm{e}}(\text { crit. }) \times \mathrm{K}_{\text {pos. }}\left(r_{\mathrm{p}_{\text {min. }}}-r_{0}\right)^{\frac{1}{4}} C_{\mathrm{b}}{ }^{-\frac{3}{8}} \\
& F_{\mathrm{e}}(\text { crit. })=\left\{D_{\mathrm{a}}{ }^{4}+\left(1-D_{\mathrm{a}}\right)^{4}\left(\frac{t_{\text {max. }}}{d_{1}}\right)^{1.5}\right\}^{-\frac{1}{4}}
\end{aligned}
$$

In these equations, $r_{\mathrm{p}_{\text {min. }}}, r_{0}$ and $t_{\text {max. }}$ are the values in meters. $d_{1}=t_{\text {max. }}-0.006(\mathrm{~m})$ in the present study. $F_{\mathrm{e}}$ (crit.) is the critical value of $F_{\mathrm{e}}$ obtained with $r_{\mathrm{p}_{\text {min. }}}$ and $t_{\text {max. }}$.

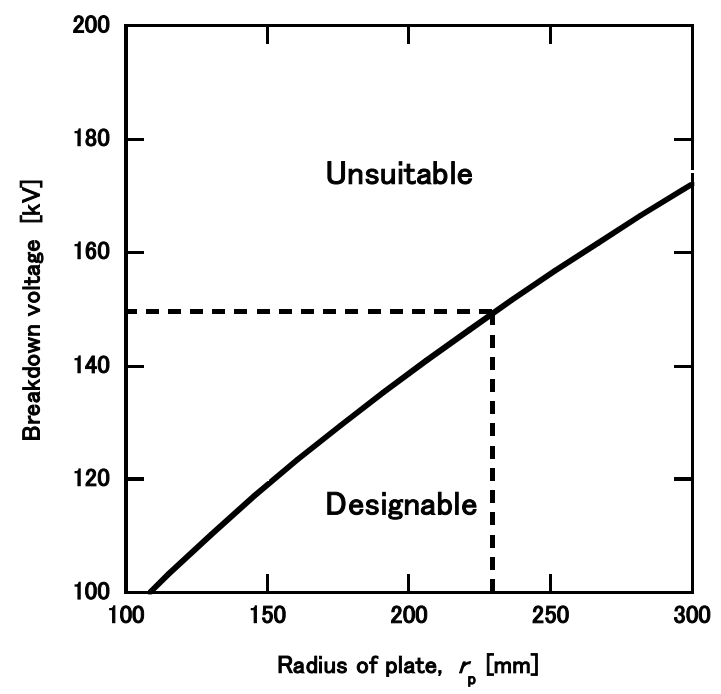

Figure 11. Critical conditions of the surface breakdown voltage: the relation between the surface breakdown voltage and the minimum radius of the plate. Broken lines indicate an example of surface insulation design when lightning impulse withstand voltage (LIWV) of $150 \mathrm{kV}$ is given.

The calculation results are shown in Figure 11 as a relation between the surface breakdown voltage and the radius of the plate $r_{\mathrm{p}}$. The curve in Figure 11 shows the critical value of the surface breakdown voltage and $r_{\mathrm{p}}$ corresponding to the critical value of the surface breakdown voltage $B D V_{\text {peak }}$ (crit.) is the minimum radius of the plate $r_{\mathrm{p}_{\text {min }}}$. The value of the thickness corresponding to the critical value of the surface breakdown voltage $B D V_{\text {peak }}$ (crit.) can be found from Figure 10 .

When an insulation level is given, the minimum radius of the epoxy resin plate can be found from Figure 11. And the value of the thickness of the plate can be found from Figure 10. The value of the thickness is the maximum value. The process of surface insulation design when lightning impulse withstand voltage (LIWV) is $150 \mathrm{kV}$ for example is taken into account. From Figure 11, it is found that the minimum radius of epoxy resin plate is $230 \mathrm{~mm}$. Then, the maximum thickness of the plate becomes $30 \mathrm{~mm}$ for the radius of the plate of $230 \mathrm{~mm}$, from Figure 10. Therefore, the optimum values of the radius and the thickness are decided $230 \mathrm{~mm}$ and $30 \mathrm{~mm}$. The optimum radius of embedded electrode can be calculated using equation (12).

\section{CONCLUSION}

Related to the optimum design for the solid insulated switchgear (SIS) developed as substitution of medium voltage $\mathrm{SF}_{6}$ gas insulated switchgear, the authors examined the surface breakdown characteristics based on the estimation method proposed previously. The authors examined the divided voltage ratio, the radius of the embedded electrode, the enhancement factor of the surface insulation strength, the peak value of the surface breakdown voltage and others. As a result, following various characteristics were obtained under the present conditions. In addition, the criteria for the optimum insulation design under the present conditions were clarified.

(1) The surface breakdown voltage (BDV) has a peak and the radius of the embedded electrode corresponding to the peak value of BDV is determined uniquely when the radius and the thickness of the epoxy resin plate are given.

(2) The divided voltage ratio is almost constant regardless of the radius of the embedded electrode except for its small region. It varies with the thickness of the plate independent of the radius of the plate.

(3) The enhancement factor of the surface insulation strength varies with the thickness of the plate.

(4) The radius of the embedded electrode has a minimum value and the minimum value varies with the thickness of the plate.

(5) The peak value of the surface breakdown voltage for given radius of the plate increases with the thickness of the plate and takes the critical value.

(6) The relation between the minimum radius and the maximum thickness of the plate is uniquely decided.

(7) The minimum radius of the plate for the optimum insulation design is found from the critical values of the surface breakdown voltage. The thickness of the plate is decided from the relation between the minimum radius and the maximum thickness of the plate.

The authors believe that the results obtained in the present study are helpful for the design of the solid/gas composite insulation system. However, the criteria can be used just for the present condition. Note that the criteria will vary with the thickness of the epoxy resin between the embedded electrode and the ground and the thickness of the embedded electrode.

\section{REFERENCES}

[1] T. Shioiri, J. Sato, T. Ozaki, O. Sakaguchi, T. Kamikawaji, M. Miyagawa, M. Homma and K. Suzuki, "Insulation Technology for Medium Voltage Solid Insulated Switchgear", IEEE Conf. Electr. Insul. Dielectr. Phenomena, pp.341-344, 2003.

[2] J. Sato, O. Sakaguchi, N. Kubota, S. Makishima, S. Kinoshita, T. Shioiri, T. Yoshida, M. Miyagawa, M. Homma and E. Kaneko, "New Technology for Medium Voltage Solid Insulated Switchgear", IEEE PES T\&D Conf., Vol.3, pp.1791-1796, 2002.

[3] T. Rokunohe, Y. Yagihashi, K. Aoyagi, T. Oomori and F. Endo: "Development of $\mathrm{SF}_{6}$-Free $72.5 \mathrm{kV}$ GIS", IEEE Trans. Power Delivery, Vol. 22, No. 3 , pp.1869-1876, 2007. 
[4] T. Yamashita, K. Iwanaga, T. Furusato, H. Koreeda, T. Fujishima and J. Sato, "Improvement of Insulation Performance of Solid/Gas Composite Insulation with Embedded Electrode", IEEE Trans. Dielect. Electr. Insul. Vol. 23, pp.787-794, 2016.

[5] T. Yamashita, K. Iwanaga, T. Furusato, H. Koreeda, T. Fujishima, N. Asari and J. Sato: "Estimation of Surface Breakdown Voltage of Solid/Gas Composite Insulation with Embedded Electrode", IEEE Trans. Dielect. Electr. Insul. Vol.23,.pp.3026-3033, 2016.

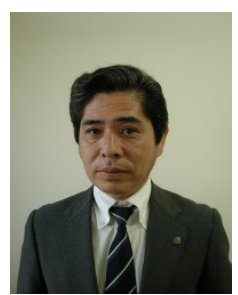

Takahiko Yamashita (M’00) was born in 1957 in Fukuoka, Japan. He received B.E., M.E. and D.E. degrees from Kyushu University in 1980, 1982 and 1985, respectively. He has been working in Nagasaki University, Nagasaki, Japan since 1985. He is a Professor of the Graduate School of Engineering and a Vice President of Nagasaki University. He is a senior member of Institute of Electrical Engineers Japan.

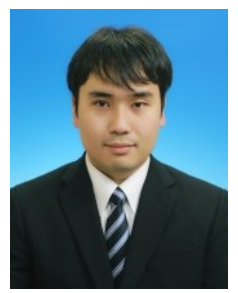

Kazuhisa Iwanaga was born in Nagasaki, Japan, in 1991. He received B.S., degree from Nagasaki University, Nagasaki Japan, in 2014, where he is currently pursuing the M.S. degree with the Graduate School of Engineering. He is mainly engaged in the development of gas/solid composite insulation systems under high stress conditions.

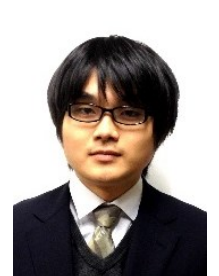

Tomohiro Furusato (M'11) was born in Kagoshima, Japan, in 1988. He received B.S., M.S., and Ph.D. degrees from Kumamoto University, Kumamoto Japan, in 2011, 2012, and 2014, respectively. He was with the Japan Society for the Promotion of Science, Kumamoto University, from 2013 to 2014, as a Research Fellow. Since 2014, he has been an Assistant Professor with the Graduate School of Engineering, Nagasaki University, Nagasaki, Japan. His research interests are pulsed-power, creeping discharge, and discharge phenomena in supercritical fluids.

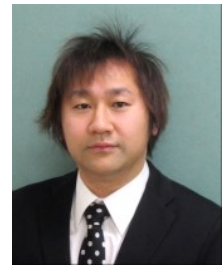

Naoki Asari was born in 1980 in Japan. He received B.S. and M.S. in electrical engineering from Saitama University in 2003 and 2005, respectively. He joined Toshiba Corporation in 2005. He is now with the switchgear and sensing system technology group. He has been engaged in research on insulation technology for switchgear and vacuum interrupter.

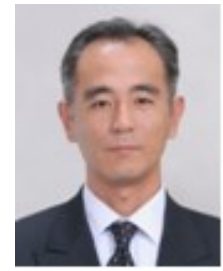

Junichi Sato was born in 1967 in Fukuoka, Japan. He received B.E., M.E. and D.E. degrees from Nagasaki University in 1990, 1992 and 2011, respectively. He has been working in Toshiba Corporation, Tokyo, Japan since 1992. He belongs to the power and industrial systems research and development center, Toshiba Corporation. His research interests are solid and solid/gas composite insulation systems, vacuum interrupter and solid insulation switchgear. 